\title{
Complete relativistic equation of state for neutron stars
}

\author{
H. Shen* \\ CCAST (World Laboratory), P.O. Box 8730, Beijing 100080, China \\ Department of Physics, Nankai University, Tianjin 300071, China ${ }^{\dagger}$ \\ Center of Theoretical Nuclear Physics, National Laboratory of Heavy Ion Accelerator, Lanzhou \\ 730000, China \\ Institute of Theoretical Physics, Beijing 100080, China
}

\begin{abstract}
We construct the equation of state (EOS) in a wide density range for neutron stars using the relativistic mean field theory. The properties of neutron star matter with both uniform and non-uniform distributions are studied consistently. The inclusion of hyperons considerably softens the EOS at high densities. The Thomas-Fermi approximation is used to describe the non-uniform matter, which is composed of a lattice of heavy nuclei. The phase transition from uniform matter to non-uniform matter occurs around $0.06 \mathrm{fm}^{-3}$, and the free neutrons drip out of nuclei at about $2.4 \times 10^{-4} \mathrm{fm}^{-3}$. We apply the resulting EOS to investigate the neutron star properties such as maximum mass and composition of neutron stars.
\end{abstract}

PACS numbers: 26.60.+c, 24.10.Jv, 21.65.+f

*Electronic address: songtc@public.tpt.tj.cn

${ }^{\dagger}$ Mailing address 


\section{INTRODUCTION}

The properties of neutron stars are mainly determined by the equation of state (EOS) of neutron star matter, which is charge neutral matter in $\beta$-equilibrium. A comprehensive description of neutron stars should include not only the interior region but also the inner and outer crusts, therefore, the EOS for neutron stars is required to cover a wide density range. For the EOS at high densities, there are many efforts based on both non-relativistic and relativistic approaches, which discussed several possible mechanisms to soften the EOS at high densities, e.q., by hyperons, kaon condensates, or even quark phases [1] 3]. When the density lower to $10^{14} \mathrm{~g} / \mathrm{cm}^{3}$, some heavy nuclei may be formed and matter becomes inhomogeneous. There are a few works based on non-relativistic models describing the EOS at low densities where the heavy nuclei exist [4 [6]. Most studies of neutron stars are using the composite EOS, which is constructed by connecting the EOS at high densities to the one at low densities [7 9]. Even though the EOS at high densities are based on various relativistic many body theories, it has to be combined with some non-relativistic EOS at low densities. The differences in the models used in the different density ranges usually lead to some discontinuity and inconsistency in the composite EOS. Therefore, it is very interesting to construct the EOS in the whole density range within the same framework.

In this paper, we provide a complete relativistic EOS for the studies of neutron stars, which is based on the relativistic mean field (RMF) theory. The RMF theory has been quite successfully and widely used for the description of nuclear matter and finite nuclei [10 12]. We study the properties of dense matter with both uniform and non-uniform distributions in the RMF framework adopting the parameter set TM1, which is known to provide excellent properties of the ground states of heavy nuclei including unstable nuclei [13]. The RMF theory with the TM1 parameter set was also shown to reproduce satisfactory agreement with experimental data in the studies of the nuclei with deformed configuration and the giant resonances within the RPA formalism 14 16. At high densities, hyperons may appear as new degrees of freedom through the weak interaction, the neutron star matter is then 
composed of neutrons, protons, hyperons, electrons, and muons in $\beta$-equilibrium. For the non-uniform matter at low densities, we perform the Thomas-Fermi calculation, in which the RMF results are taken as its input. The non-uniform matter is assumed to be composed of a lattice of spherical nuclei immersed in an electron gas with (or without) free neutrons dripping out of nuclei [17,[18]. The optimal state at each density is determined by minimizing the energy density with respect to the independent parameters in the model. The phase transition from non-uniform matter to uniform matter takes place around $10^{14} \mathrm{~g} / \mathrm{cm}^{3}$. The same method (but without the inclusion of hyperons) has been used to work out the equation of state at finite temperature with various proton fractions for the use of supernova simulations [19,20.

This paper is arranged as follows. In Sec. II, we briefly describe the RMF theory and its parameters. In Sec. III, we explain the Thomas-Fermi approximation used for the description of non-uniform matter. The resulting EOS in the whole density range is shown and discussed in Sec. IV. We apply the relativistic EOS to study the constitution and structure of neutron stars in Sec. V. The conclusion is presented in Sec. VI.

\section{RELATIVISTIC MEAN FIELD THEORY}

We briefly explain the RMF theory used to describe the uniform matter. In the RMF theory, baryons interact via the exchange of mesons. The baryons considered in the present calculation include nucleons $(n$ and $p)$ and hyperons $(\Lambda, \Sigma, \Xi)$. The exchanged mesons consist of isoscalar scalar and vector mesons ( $\sigma$ and $\omega)$, isovector vector meson $(\rho)$, and two strange mesons $\left(\sigma^{*}\right.$ and $\left.\phi\right)$ which couple only to hyperons. The total Lagrangian density of neutron star matter, in the mean field approximation, can be written as

$$
\begin{aligned}
\mathcal{L}= & \sum_{B} \bar{\psi}_{B}\left[i \gamma_{\mu} \partial^{\mu}-\left(m_{B}-g_{\sigma B} \sigma-g_{\sigma^{*} B} \sigma^{*}\right)-\left(g_{\omega B} \omega+g_{\phi B} \phi+g_{\rho B} \tau_{3} \rho\right) \gamma^{0}\right] \psi_{B} \\
& -\frac{1}{2} m_{\sigma}^{2} \sigma^{2}+\frac{1}{3} g_{2} \sigma^{3}-\frac{1}{4} g_{3} \sigma^{4}+\frac{1}{2} m_{\omega}^{2} \omega^{2}+\frac{1}{4} c_{3} \omega^{4}+\frac{1}{2} m_{\rho}^{2} \rho^{2} \\
& -\frac{1}{2} m_{\sigma^{*}}^{2} \sigma^{* 2}+\frac{1}{2} m_{\phi}^{2} \phi^{2}+\sum_{l} \bar{\psi}_{l}\left(i \gamma_{\mu} \partial^{\mu}-m_{l}\right) \psi_{l}
\end{aligned}
$$


where the sum on $B$ is over all the charge states of the baryon octet $\left(p, n, \Lambda, \Sigma^{+}, \Sigma^{0}, \Sigma^{-}, \Xi^{0}\right.$, $\left.\Xi^{-}\right)$, and the sum on $l$ is over the electrons and muons $\left(e^{-}\right.$and $\left.\mu^{-}\right)$. The meson mean fields are denoted as $\sigma, \omega, \rho, \sigma^{*}$, and $\phi$. The inclusion of the non-linear $\sigma$ and $\omega$ terms is essential to reproduce the feature of the relativistic Brueckner-Hartree-Fock theory and satisfactory properties of finite nuclei [13]. We adopt the TM1 parameter set for the meson-nucleon couplings and the self-coupling constants and some masses, which was determined in Ref. 13 by reproducing the properties of finite nuclei in the wide mass range in the periodic table including neutron-rich nuclei. The RMF theory with the TM1 parameter set was also shown to reproduce satisfactory agreement with experimental data in the studies of the nuclei with deformed configuration and the giant resonances within the RPA formalism [14] [16]. The hyperon masses are taken to be $m_{\Lambda}=1116 \mathrm{MeV}, m_{\Sigma}=1193 \mathrm{MeV}$, and $m_{\Xi}=1313 \mathrm{MeV}$, while the strange meson masses are $m_{\sigma^{*}}=975 \mathrm{MeV}$ and $m_{\phi}=1020 \mathrm{MeV}$ [3]. As for the hyperon couplings, we employ the following relations derived from the quark model

$$
\begin{aligned}
& \frac{1}{3} g_{\sigma N}=\frac{1}{2} g_{\sigma \Lambda}=\frac{1}{2} g_{\sigma \Sigma}=g_{\sigma \Xi} \\
& \frac{1}{3} g_{\omega N}=\frac{1}{2} g_{\omega \Lambda}=\frac{1}{2} g_{\omega \Sigma}=g_{\omega \Xi}, \\
& g_{\rho N}=\frac{1}{2} g_{\rho \Sigma}=g_{\rho \Xi}, \quad g_{\rho \Lambda}=0, \\
& 2 g_{\sigma^{*} \Lambda}=2 g_{\sigma^{*} \Sigma}=g_{\sigma^{*} \Xi}=\frac{2 \sqrt{2}}{3} g_{\sigma N}, \quad g_{\sigma^{*} N}=0, \\
& 2 g_{\phi \Lambda}=2 g_{\phi \Sigma}=g_{\phi \Xi}=\frac{2 \sqrt{2}}{3} g_{\omega N}, \quad g_{\phi N}=0 .
\end{aligned}
$$

In the RMF theory, the meson fields are treated as classical fields, and the field operators are replaced by their expectation values. The meson field equations in uniform matter are given by

$$
\begin{aligned}
& m_{\sigma}^{2} \sigma-g_{2} \sigma^{2}+g_{3} \sigma^{3}=\sum_{B} g_{\sigma B} \frac{2 J_{B}+1}{2 \pi^{2}} \int_{0}^{k_{B}} \frac{m_{B}^{*}}{\sqrt{k^{2}+m_{B}^{* 2}}} k^{2} d k \\
& m_{\omega}^{2} \omega+c_{3} \omega^{3}=\sum_{B} g_{\omega B}\left(2 J_{B}+1\right) k_{B}^{3} /\left(6 \pi^{2}\right) \\
& m_{\rho}^{2} \rho=\sum_{B} g_{\rho B} I_{3 B}\left(2 J_{B}+1\right) k_{B}^{3} /\left(6 \pi^{2}\right), \\
& m_{\sigma^{*}}^{2} \sigma^{*}=\sum_{B} g_{\sigma^{*} B} \frac{2 J_{B}+1}{2 \pi^{2}} \int_{0}^{k_{B}} \frac{m_{B}^{*}}{\sqrt{k^{2}+m_{B}^{* 2}}} k^{2} d k
\end{aligned}
$$




$$
m_{\phi}^{2} \phi=\sum_{B} g_{\phi B}\left(2 J_{B}+1\right) k_{B}^{3} /\left(6 \pi^{2}\right)
$$

where $m_{B}^{*}=m_{B}-g_{\sigma B} \sigma-g_{\sigma^{*} B} \sigma^{*}$ is the effective mass of the baryon species $B$, and $k_{B}$ is its Fermi momentum. $J_{B}$ and $I_{3 B}$ denote the spin and the isospin projection of baryon $B$.

For neutron star matter with uniform distributions, the composition is determined by the requirements of charge neutrality and $\beta$-equilibrium conditions. Considering the baryon octet and leptons included in the present calculation, the $\beta$-equilibrium conditions, without trapped neutrinos, can be clearly expressed by

$$
\begin{aligned}
& \mu_{p}=\mu_{\Sigma^{+}}=\mu_{n}-\mu_{e}, \\
& \mu_{\Lambda}=\mu_{\Sigma^{0}}=\mu_{\Xi^{0}}=\mu_{n}, \\
& \mu_{\Sigma^{-}}=\mu_{\Xi^{-}}=\mu_{n}+\mu_{e}, \\
& \mu_{\mu}=\mu_{e}
\end{aligned}
$$

where $\mu_{i}$ is the chemical potential of species $i$. At zero temperature, the chemical potentials of baryon $B$ and lepton $l$ are given by

$$
\begin{aligned}
& \mu_{B}=\sqrt{k_{B}^{2}+m_{B}^{* 2}}+g_{\omega B} \omega+g_{\phi B} \phi+g_{\rho B} \tau_{3} \rho, \\
& \mu_{l}=\sqrt{k_{l}^{2}+m_{l}^{* 2}} .
\end{aligned}
$$

The charge neutrality condition has the following form:

$$
n_{p}+n_{\Sigma^{+}}=n_{e}+n_{\mu}+n_{\Sigma^{-}}+n_{\Xi^{-}},
$$

where $n_{i}=k_{i}^{3} / 3 \pi^{2}$ is the number density of species $i$. Then, the total baryon density is $n_{B}=n_{n}+n_{p}+n_{\Lambda}+n_{\Sigma^{-}}+n_{\Sigma^{0}}+n_{\Sigma^{+}}+n_{\Xi^{-}}+n_{\Xi^{0}}$. We solve the coupled equations (3)-(7), (8), and (11) self-consistently at a given baryon density $n_{B}$. The total energy density and pressure of the uniform matter are given by

$$
\begin{aligned}
\varepsilon= & \sum_{B} \frac{2 J_{B}+1}{2 \pi^{2}} \int_{0}^{k_{B}} \sqrt{k^{2}+m_{B}^{* 2}} k^{2} d k+\frac{1}{2} m_{\sigma}^{2} \sigma^{2}-\frac{1}{3} g_{2} \sigma^{3}+\frac{1}{4} g_{3} \sigma^{4} \\
& +\frac{1}{2} m_{\omega}^{2} \omega^{2}+\frac{3}{4} c_{3} \omega^{4}+\frac{1}{2} m_{\rho}^{2} \rho^{2}+\frac{1}{2} m_{\sigma^{*}}^{2} \sigma^{* 2}+\frac{1}{2} m_{\phi}^{2} \phi^{2} \\
& +\sum_{l} \frac{1}{\pi^{2}} \int_{0}^{k_{l}} \sqrt{k^{2}+m_{l}^{2}} k^{2} d k
\end{aligned}
$$




$$
\begin{aligned}
P= & \frac{1}{3} \sum_{B} \frac{2 J_{B}+1}{2 \pi^{2}} \int_{0}^{k_{B}} \frac{k^{4} d k}{\sqrt{k^{2}+m_{B}^{* 2}}}-\frac{1}{2} m_{\sigma}^{2} \sigma^{2}+\frac{1}{3} g_{2} \sigma^{3}-\frac{1}{4} g_{3} \sigma^{4} \\
& +\frac{1}{2} m_{\omega}^{2} \omega^{2}+\frac{1}{4} c_{3} \omega^{4}+\frac{1}{2} m_{\rho}^{2} \rho^{2}-\frac{1}{2} m_{\sigma^{*}}^{2} \sigma^{* 2}+\frac{1}{2} m_{\phi}^{2} \phi^{2} \\
& +\frac{1}{3} \sum_{l} \frac{1}{\pi^{2}} \int_{0}^{k_{l}} \frac{k^{4} d k}{\sqrt{k^{2}+m_{l}^{2}}} .
\end{aligned}
$$

\section{THOMAS-FERMI APPROXIMATION}

In the low density range, where heavy nuclei exist, we perform the Thomas-Fermi calculation based on the work done by Oyamatsu [17]. In this approximation, the non-uniform matter can be modeled as a lattice of nuclei immersed in a vapor of neutrons and electrons. At lower density, there is no neutron dripping out of nuclei. We assume that each heavy spherical nucleus is located in the center of a charge-neutral cell consisting of a vapor of neutrons and electrons. The nuclei form a body-centered-cubic (BCC) lattice to minimize the Coulomb lattice energy. It is useful to introduce the Wigner-Seitz cell to simplify the energy of the unit cell. The Wigner-Seitz cell is a sphere whose volume is the same as the unit cell in the BCC lattice.

We assume the nucleon distribution functions $n_{i}(r)$ ( $i=n$ for neutron, $i=p$ for proton) in the Wigner-Seitz cell as

$$
n_{i}(r)=\left\{\begin{array}{ll}
\left(n_{i}^{\text {in }}-n_{i}^{\text {out }}\right)\left[1-\left(\frac{r}{R_{i}}\right)^{t_{i}}\right]^{3}+n_{i}^{\text {out }}, & 0 \leq r \leq R_{i} \\
n_{i}^{\text {out }}, & R_{i} \leq r \leq R_{\text {cell }}
\end{array},\right.
$$

where $r$ represents the distance from the center of the nucleus, and $R_{\text {cell }}$ is the radius of the

Wigner-Seitz cell defined by the relation, $V_{\text {cell }}=\frac{4 \pi}{3} R_{\text {cell }}^{3}=a^{3}$ ( $a$ is the lattice constant). The parameters $R_{i}$ and $t_{i}$ determine the boundary and the relative surface thickness of the heavy nucleus. $R_{n}$ and $t_{n}$ may be a little different from $R_{p}$ and $t_{p}$ due to the additional neutrons forming a neutron skin in the surface region. For neutron star matter at a given average density of baryons, $n_{B}=\int_{\text {cell }}\left[n_{n}(r)+n_{p}(r)\right] d^{3} r / V_{\text {cell }}$, there are only seven independent parameters among the eight variables: $a, n_{n}^{\text {in }}, n_{n}^{\text {out }}, R_{n}, t_{n}, n_{p}^{i n}, R_{p}, t_{p}$. The optimal state is 
determined by minimizing the average energy density, $\varepsilon=E_{\text {cell }} / V_{\text {cell }}$, with respect to those independent parameters.

The total energy per cell, $E_{\text {cell }}$, can be written as

$$
E_{\text {cell }}=E_{\text {bulk }}+E_{s}+E_{C}+E_{e} .
$$

Here the bulk energy of baryons, $E_{b u l k}$, is calculated by

$$
E_{\text {bulk }}=\int_{\text {cell }} \varepsilon_{R M F}\left(n_{n}(r), n_{p}(r)\right) d^{3} r
$$

where $\varepsilon_{R M F}$ is the energy density in the RMF theory as the functional of the neutron density $n_{n}$ and the proton density $n_{p}$. As the input in the Thomas-Fermi calculation, $\varepsilon_{R M F}$ at each radius $r$ is calculated in the RMF theory for uniform matter with the corresponding densities $n_{n}$ and $n_{p}$. The surface energy term $E_{s}$ due to the inhomogeneity of nucleon distribution is given by,

$$
E_{s}=\int_{\text {cell }} F_{0}\left|\nabla\left(n_{n}(r)+n_{p}(r)\right)\right|^{2} d^{3} r
$$

where the parameter $F_{0}=70 \mathrm{MeV} \cdot \mathrm{fm}^{5}$ is determined by doing the Thomas-Fermi calculations of finite nuclei as described in the appendix in Ref. [17]. The Coulomb energy per cell $E_{C}$ is calculated using the Wigner-Seitz approximation with an added correction term for the BCC lattice:

$$
E_{C}=\frac{1}{2} \int_{\text {cell }} e\left[n_{p}(r)-n_{e}\right] \phi(r) d^{3} r+\triangle E_{C}
$$

where $\phi(r)$ stands for the electrostatic potential calculated in the Wigner-Seitz approximation, $\triangle E_{C}=C_{B C C}(Z e)^{2} / a$ is the correction term for the BCC lattice as given in Ref. [17]. $n_{e}$ is the electron number density of uniform electron gas, which can be determined by the charge neutrality condition as $n_{e}=Z / V_{\text {cell }}$ ( $Z$ denotes the proton number per cell). The last term in Eq. (15), $E_{e}$, is the kinetic energy of electrons, which is given by

$$
E_{e}=\frac{1}{\pi^{2}} \int_{0}^{k_{e}} \sqrt{k^{2}+m_{e}^{2}} k^{2} d k
$$


where $k_{e}=\left(3 \pi^{2} n_{e}\right)^{1 / 3}$ is the Fermi momentum of electrons.

For each baryon density $n_{B}$, we minimize the average energy density $\varepsilon$ of non-uniform matter with respect to the independent parameters in the Thomas-Fermi approximation. At some higher densities, the heavy nuclei dissolve and the matter becomes homogeneous. We determine the density, at which the phase transition takes place, by comparing the energy density of non-uniform matter with the one of uniform matter.

\section{PROPERTIES OF NEUTRON STAR MATTER}

In this section, we present the resulting EOS of neutron star matter in the density range from $10^{-7}$ to $1.2 \mathrm{fm}^{-3}$. At low densities where heavy nuclei exist, the non-uniform matter is described by the Thomas-Fermi approximation, in which the optimal state is determined by minimizing the average energy density with respect to its independent parameters. For the density below $\sim 2.4 \times 10^{-4} \mathrm{fm}^{-3}$, the nucleons form the optimal nuclei and those nuclei build a BCC lattice with uniform electron gas. It is found that the neutrons begin to drip out from nuclei at $n_{B} \sim 2.4 \times 10^{-4} \mathrm{fm}^{-3}$, then there is a neutron gas in addition to the electron gas. In Fig. 1 1 we show the neutron and the proton distributions along the straight line joining the centers of the nearest nuclei in the BCC lattice at the average baryon densities $n_{B}=0.0001,0.001,0.01,0.05 \mathrm{fm}^{-3}$. As the density increases, the optimal nuclei become closer and more neutron rich. At $n_{B} \sim 0.06 \mathrm{fm}^{-3}$, the nuclei dissolve and the optimal state is a uniform matter consisting of neutrons, protons, and electrons in $\beta$-equilibrium. When the electron chemical potential exceeds the rest mass of the muon (at $n_{B} \approx 0.11 \mathrm{fm}^{-3}$ ), it becomes energetically favorable to convert the electrons at the Fermi surface into muons, then the muons appear with the chemical equilibrium condition $\mu_{e}=\mu_{\mu}$. Hyperons appear

at higher densities $\left(n_{B} \gtrsim 0.27 \mathrm{fm}^{-3}\right)$. In Fig. 2 we show the fraction of species $i, Y_{i}=n_{i} / n_{B}$, as a function of the total baryon density $n_{B}$. The composition of uniform neutron star matter is calculated by solving the coupled equations (3)-(7), (8), and (11). The threshold density for a hyperon species is determined not only by its charge and mass but also by the meson 
mean fields, which are shown in Fig. 3 as functions of baryon density. In the present calculation, $\Sigma^{-}$is the first hyperon which appears at $n_{B} \approx 0.27 \mathrm{fm}^{-3}$, while $\Lambda$ has almost the same threshold density $\left(n_{B} \approx 0.29 \mathrm{fm}^{-3}\right)$. It is partly because the negative charge is much more favorable, even though $\Sigma^{-}$has somewhat larger mass compared with the mass of $\Lambda$. The other hyperons, $\Sigma^{0}, \Sigma^{+}, \Xi^{-}$, and $\Xi^{0}$, appear one by one at higher densities $\left(n_{B} \approx 0.57,0.72,0.84,1.17 \mathrm{fm}^{-3}\right)$. The appearance of hyperons causes some decreases of the nucleon fractions. At high densities $\left(n_{B} \gtrsim 0.7 \mathrm{fm}^{-3}\right)$, the $\Lambda$ fraction is larger than the neutron fraction. We note that the hyperon threshold densities and fractions are sensitive to the hyperon couplings, and there are quite large uncertainties in the hyperon couplings. In this work, we adopt the hyperon couplings derived from the quark model.

We display in Fig. 4 the pressure of neutron star matter as a function of energy density. The present EOS shown by the solid curve is compared with the EOS considering only the uniform matter phase (dotted curve), and it is found that the contribution from the nonuniform matter is quite large at low densities. The EOS without hyperons is also shown for comparison by dashed curve. The inclusion of hyperons considerably softens the EOS at high densities, because the conversion of nucleons to hyperons can relieve the Fermi pressure of the nucleons. In Fig. 5 we show the fraction of species $i, Y_{i}$, in neutron star matter as a function of the average baryon density $n_{B}$. It is very interesting to see the phase transitions in the wide density range. At low densities, all nucleons exist inside nuclei, therefore the fraction of the nucleons in nuclei (dot-dashed curve) is equal to one. The decrease of the electron fraction (dotted curve), which is equal to the proton fraction due to the charge neutrality, implies that the optimal nucleus becomes more and more neutron rich as the density increases. Beyond the neutron drip density $\left(n_{B} \sim 2.4 \times 10^{-4} \mathrm{fm}^{-3}\right)$, there is a increasing fraction of the free neutrons outside nuclei (solid curve), and this causes a rapid decrease of the fraction of the nucleons in nuclei (dot-dashed curve). The phase transition from non-uniform matter to uniform matter occurs at $\sim 0.06 \mathrm{fm}^{-3}$, where the heavy nuclei dissolve and the matter consists of neutrons, protons, and electrons in $\beta$-equilibrium. We note that the neutron star matter is assumed to be at zero temperature, so there is no free 
proton gas outside nuclei in the non-uniform matter phase. The muon fraction appears at $n_{B} \approx 0.11 \mathrm{fm}^{-3}$ with the charge neutrality condition $Y_{\mu}+Y_{e}=Y_{p}$. At high densities $\left(n_{B} \gtrsim 0.27 \mathrm{fm}^{-3}\right)$, the hyperon fractions appear, which have been shown more clearly in Fig. 2.

\section{NEUTRON STAR STRUCTURE}

We calculate the neutron star properties by using the relativistic EOS. The neutron star masses as functions of central baryon density are displayed in Fig. 6. It is shown that the maximum mass of the neutron stars including hyperons is around $1.6 M_{\odot}$, while it is around $2.2 M_{\odot}$ without hyperons. The neutron star mass is determined predominantly by the behavior of the EOS at high densities. The inclusion of hyperons considerably softens the EOS at high densities, therefore, results much smaller neutron star masses. The nonuniform matter, which exists in the crusts of neutron stars, has negligible contribution to the total neutron star mass, but it plays an important role in the description of the neutron star profile in the crustal region. In Fig. 7 and 8, we show the number density of the composition in the neutron stars with $M=1.6 M_{\odot}$ and $M=1.2 M_{\odot}$ respectively, as a function of radius. It is clear that the uniform matter containing the equilibrium mixture of nucleons, hyperons, and leptons exists in the internal region of the neutron star, while the non-uniform matter phase occurs only in the surface region. The neutron star with $M=1.6 M_{\odot}$ has much thinner crusts as compared to the case of the neutron star with $M=1.2 M_{\odot}$. We show in Fig. 9 the mass-radius relations using the EOS with or without hyperons. It is found that the inclusion of hyperons only influences the neutron stars having large masses $\left(M \gtrsim 1.2 M_{\odot}\right)$.

\section{CONCLUSION}

We have constructed the relativistic EOS of neutron star matter in the density range from $10^{-7}$ to $1.2 \mathrm{fm}^{-3}$. The non-uniform matter at low densities has been described by the Thomas-Fermi approximation, in which the nucleons form the optimal nuclei and those 
nuclei build a BCC lattice. The uniform matter at high densities has been studied in the RMF theory. We adopted the RMF model with the TM1 parameter set, which was demonstrated to be successful in describing the properties of nuclear matter and finite nuclei including unstable nuclei [13], and its results were taken as the input in the Thomas-Fermi calculations. Hence we have worked out consistent calculations for uniform matter and nonuniform matter. The phase transition from non-uniform matter to uniform matter is found to take place at $n_{B} \sim 0.06 \mathrm{fm}^{-3}$. At high densities $\left(n_{B} \gtrsim 0.27 \mathrm{fm}^{-3}\right)$, it is energetically favorable to convert some nucleons into hyperons via weak interactions. The inclusion of hyperons leads to a considerable softening of the EOS at high densities, since the conversion of nucleons to hyperons can relieve the Fermi pressure of the nucleons. We note that the contributions from hyperons are sensitive to the hyperon couplings, here we have adopted the hyperon couplings derived from the quark model. Presently, there exist large uncertainties in hyperon couplings. The hyperon couplings should be constrained by the experimental data of hypernuclei, but the experimental information is deficient for determining them. From the study of single $\Lambda$ hypernuclei, the quark model values of $\Lambda$ hyperon couplings usually predict overbinding of $\Lambda$ single particle energies. It seems that the quark model values of $\Lambda$ hyperon couplings lead to rather strong attraction. This might cause an earlier appearance of $\Lambda$ hyperon. A detailed investigation of the dependence of the results on the hyperon couplings is deferred to future work.

We have employed the present EOS to calculate the neutron star properties. With the appearance of hyperons, the maximum mass of neutron stars turned out to be $1.6 M_{\odot}$. It is found that the inclusion of hyperons results much smaller neutron star masses due to the softening of the EOS. The core of massive neutron stars is then composed of the equilibrium mixture of nucleons, hyperons, and leptons. The non-uniform matter exists only in the surface region, which forms quite thin crusts of neutron stars. The consideration of the non-uniform matter phase has negligible contribution to the neutron star mass, but it is essential to provide a realistic description of the neutron star structure.

The present calculations have been performed within the framework of the relativistic 
mean field approach, which is incapable to include pions explicitly. It will be possible and important to construct a complete EOS based on more microscopic theory such as the Dirac-Brueckner-Hartree-Fock approach. Especially, same approach should be employed in the treatment of both uniform matter and non-uniform matter. It is well known that the relativity plays an essential role in describing the nuclear saturation and the nuclear structure, it also brings some distinctive properties in the EOS comparing with the case in the non-relativistic framework. Therefore, it is very interesting and important to study the astrophysical phenomena such as neutron star properties using the relativistic EOS.

\section{ACKNOWLEDGMENTS}

The author would like to thank H. Toki, K. Sumiyoshi, and K. Oyamatsu for fruitful discussions and collaborations. This work was supported in part by the National Natural Science Foundation of China under contract No. 10075028 and No. 10135030. 


\section{REFERENCES}

[1] M. Prakash, I. Bombaci, M. Prakash, P.J. Ellis, J.M. Lattimer, and R. Knorren, Phys. Rep. 280, 1 (1997).

[2] H. Heiselberg and M. Hjorth-Jensen, Phys. Rep. 328, 237 (2000).

[3] S. Pal, M. Hanauske, I. Zakout, H. Stocker, and W. Greiner, Phys. Rev. C 60, 015802 (1999).

[4] G. Baym, H.A. Bethe, and C.J. Pethick, Nucl. Phys. A175, 225 (1971).

[5] J.W. Negele and D. Vautherin, Nucl. Phys. A207, 298 (1973).

[6] C.J. Pethick and D.G. Ravenhall, Annu. Rev. Nucl. Part. Sci. 45, 429 (1995).

[7] N.K. Glendenning, F. Weber, and S.A. Moszkowski, Phys. Rev. C 45, 844 (1992).

[8] P.K. Sahu, Phys. Rev. C 62, 045801 (2000).

[9] K. Schertler, C. Greiner, J. Schaffner-Bielich, and M.H. Thoma, Nucl. Phys. A677, 463 $(2000)$.

[10] B.D. Serot and J.D. Walecka, Adv. Nucl. Phys. 16, 1 (1986).

[11] Y.K. Gambhir, P. Ring, A. Thimet, Ann. Phys. 198, 132 (1990).

[12] D. Hirata, K. Sumiyoshi, B.V. Carlson, H. Toki, and I. Tanihata, Nucl. Phys. A609 , $131(1996)$.

[13] Y. Sugahara and H. Toki, Nucl. Phys. A579 , 557 (1994).

[14] D. Hirata, H. Toki, and I. Tanihata, Nucl. Phys. A589, 239 (1995).

[15] Z.Y. Ma, H. Toki, B.Q. Chen, and N. Van Giai, Prog. Theor. Phys. 98, 917 (1997).

[16] Z.Y. Ma, N. Van Giai, A. Wandelt, D. Vretenar, and P. Ring, Nucl. Phys. A686, 173 (2001). 
[17] K. Oyamatsu, Nucl. Phys. A561 , 431 (1993).

[18] K. Sumiyoshi, K. Oyamatsu, and H. Toki, Nucl. Phys. A595, 327 (1995).

[19] H. Shen, H. Toki, K. Oyamatsu, and K. Sumiyoshi, Nucl. Phys. A637, 435 (1998).

[20] H. Shen, H. Toki, K. Oyamatsu, and K. Sumiyoshi, Prog. Theor. Phys. 100, 1013 (1998). 


\section{FIGURES}

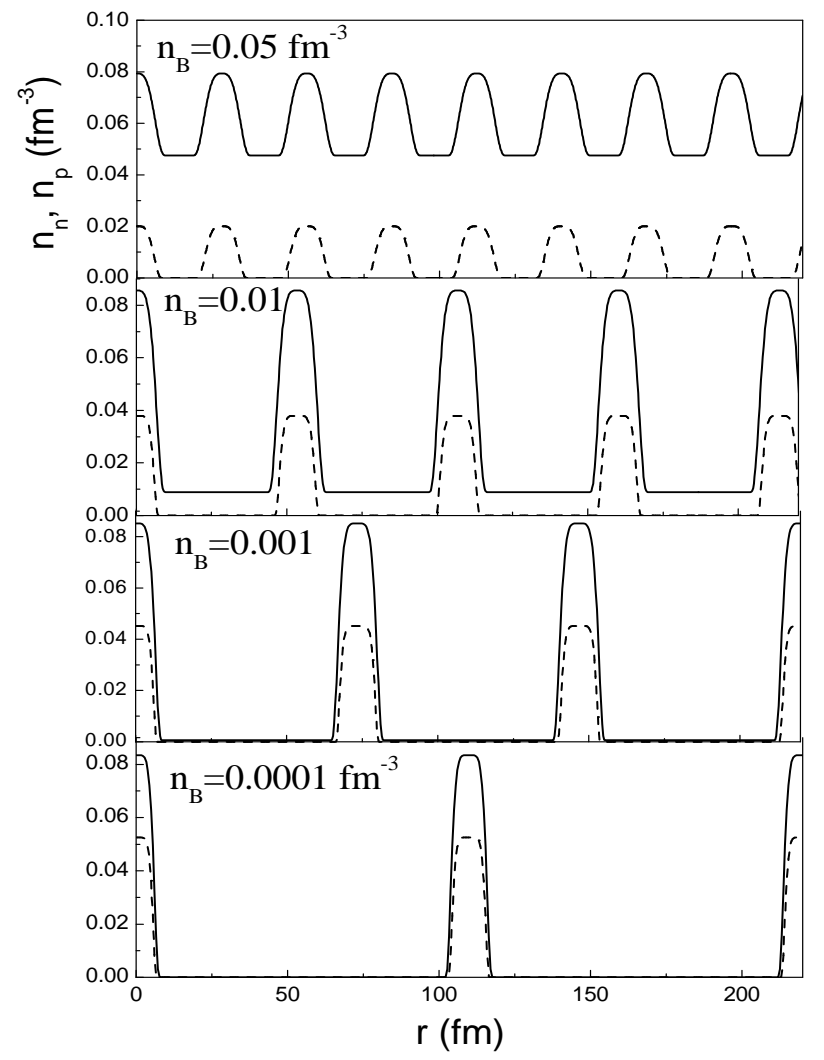

FIG. 1. The neutron distribution (solid curves) and the proton distribution (dashed curves) along the straight lines joining the centers of the nearest nuclei in the BCC lattice at the average baryon density $n_{B}=0.0001,0.001,0.01,0.05 \mathrm{fm}^{-3}$. 


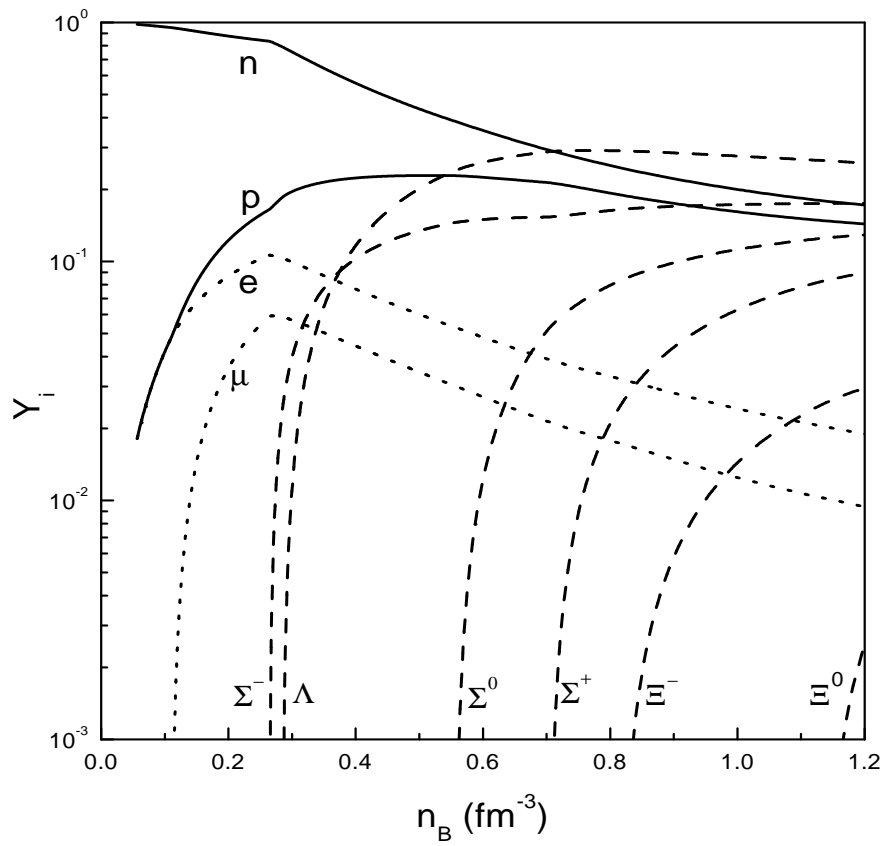

FIG. 2. The fraction of species $i, Y_{i}=n_{i} / n_{B}$, as a function of the total baryon density $n_{B}$. 


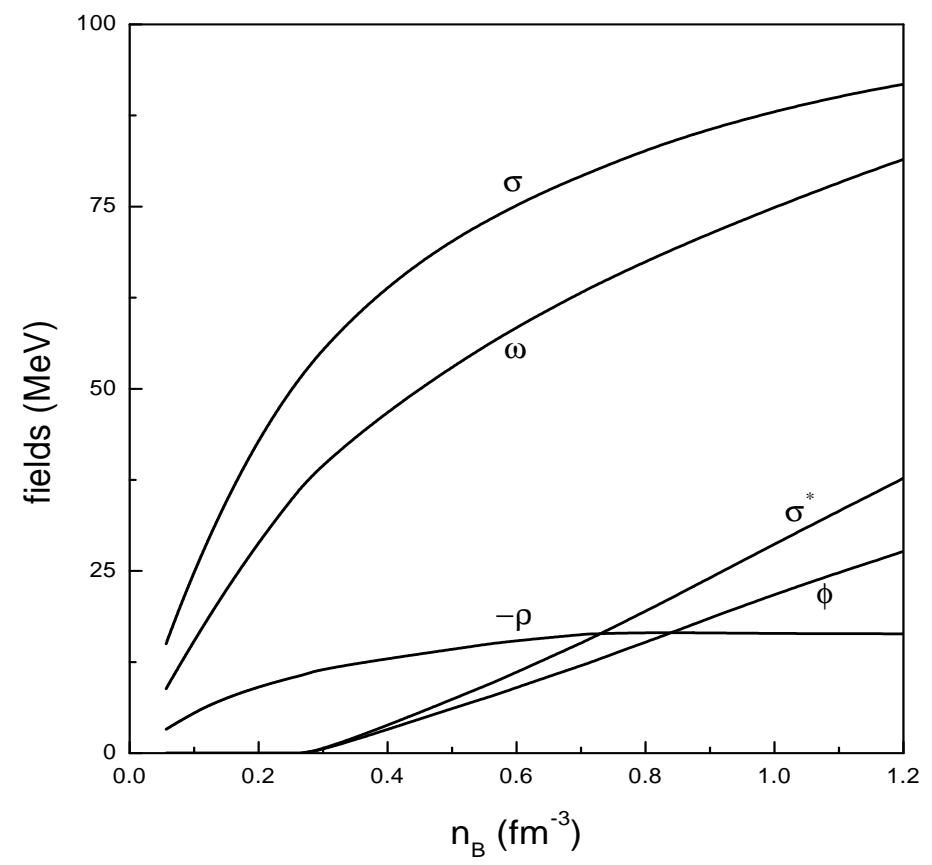

FIG. 3. The meson mean fields as functions of baryon density. 


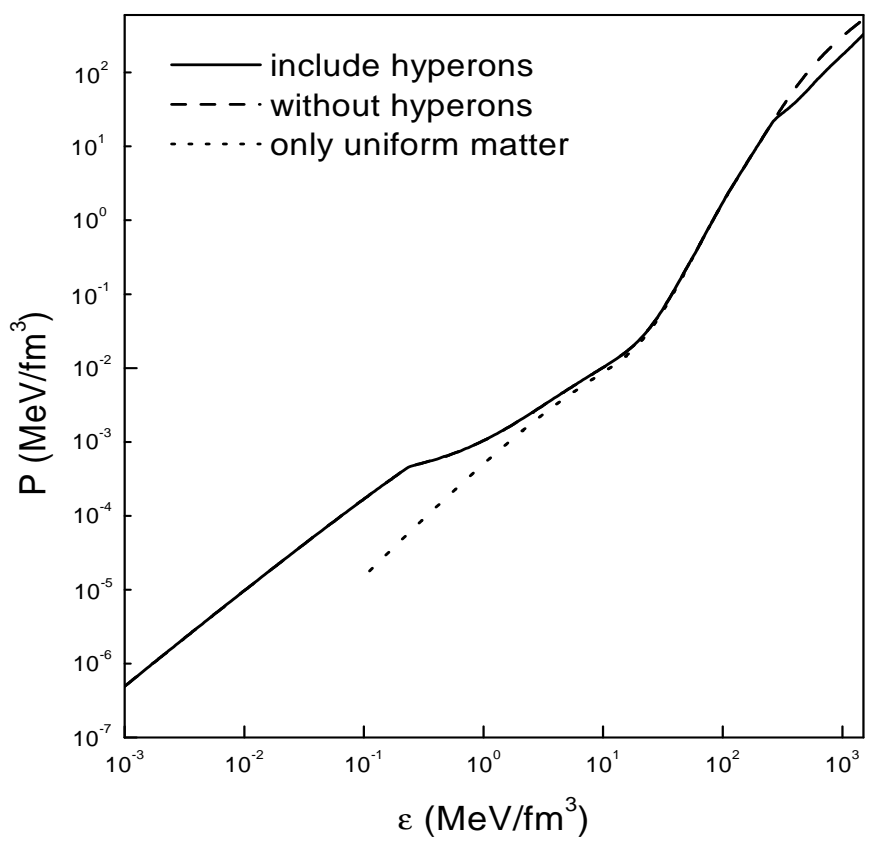

FIG. 4. The pressure $P$ versus energy density $\varepsilon$ for neutron star matter with the inclusion of hyperons (solid curve) and without hyperons (dashed curve). The EOS considering only uniform matter phase (dotted curve) is also shown for comparison. 


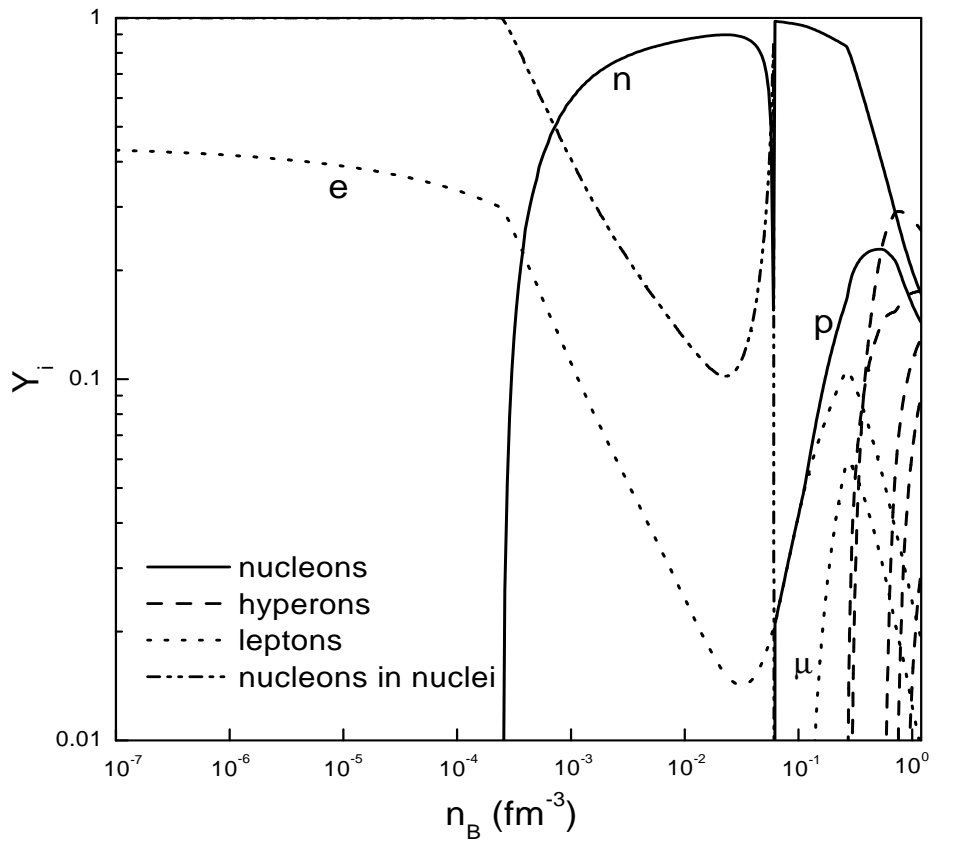

FIG. 5. The fractions of the composition in neutron star matter as functions of baryon density. 


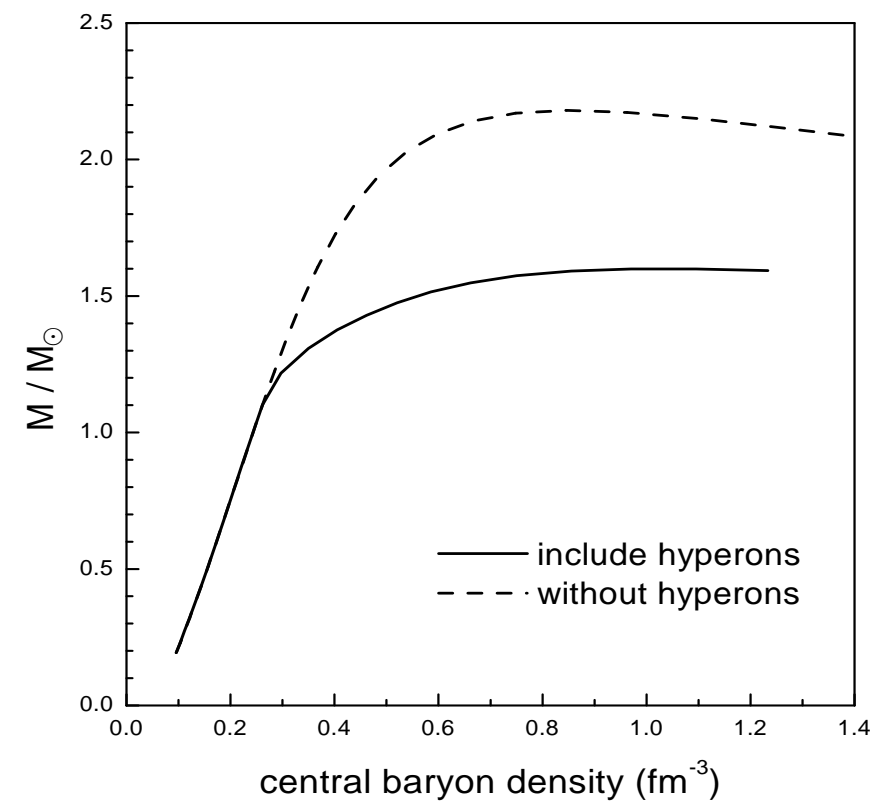

FIG. 6. The neutron star masses as functions of central baryon density 


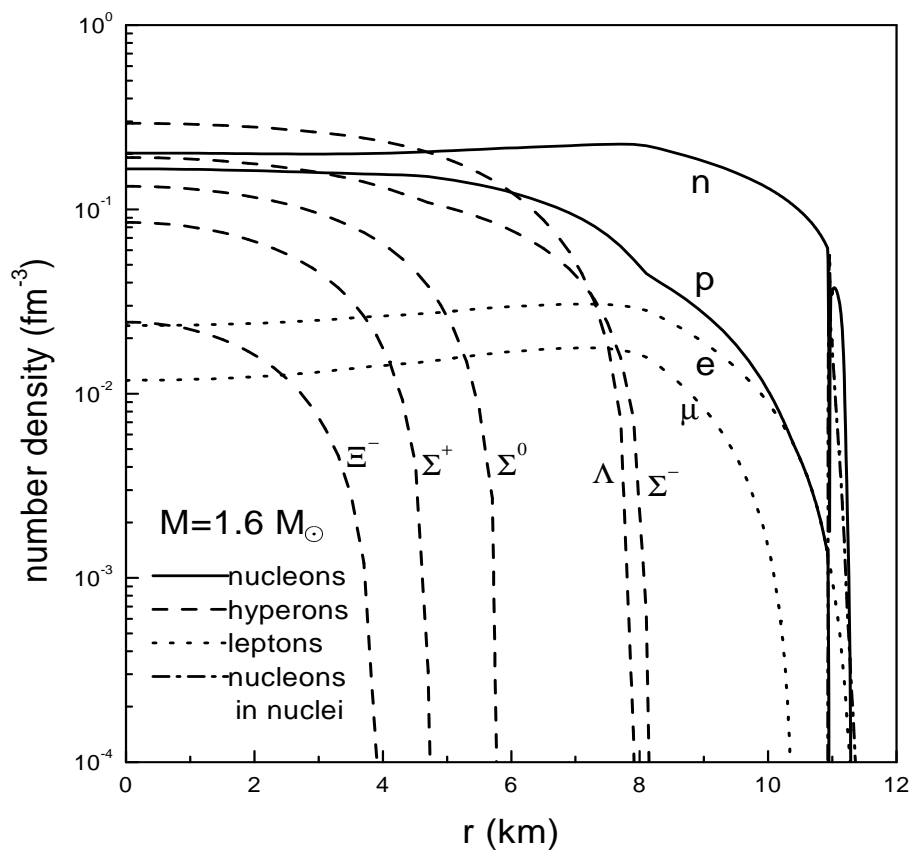

FIG. 7. The number density of the composition in the neutron star with $M=1.6 M_{\odot}$ as a function of radius $r$. 


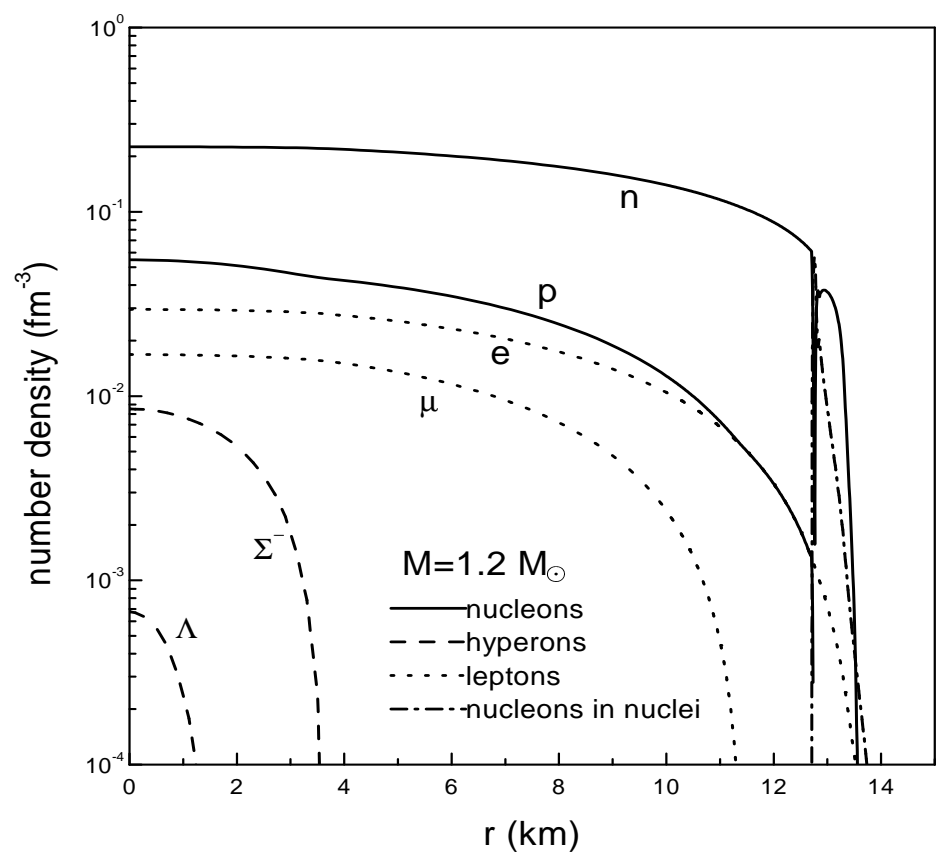

FIG. 8. Same as Fig. 7 but for $M=1.2 M_{\odot}$. 


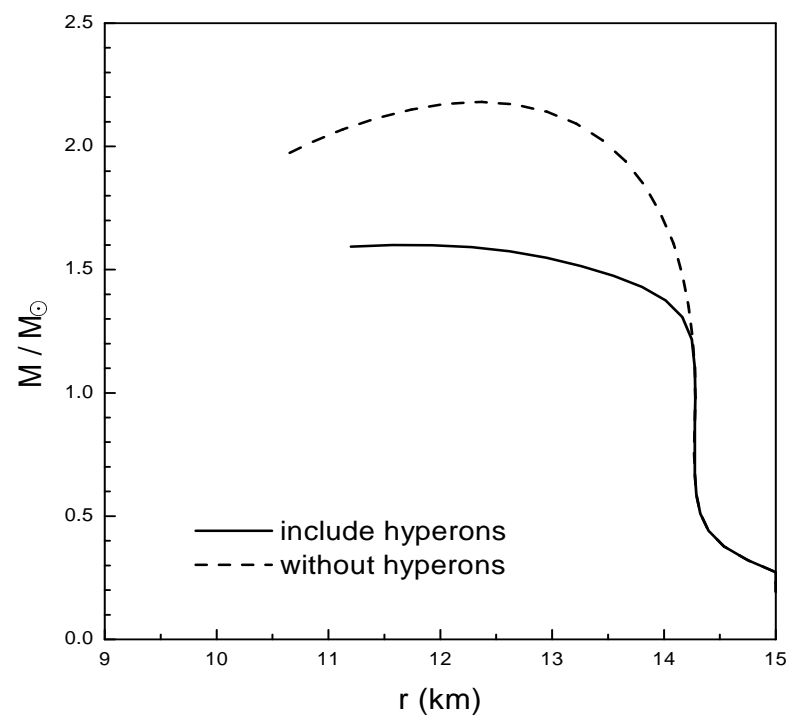

FIG. 9. The mass-radius relations for neutron stars. The solid curve shows the results with the inclusion of hyperons, while those without hyperons is plotted by the dashed curve for comparison. 